\title{
An efficient and self-adapting colour-image encryption algorithm based on chaos and interactions among multiple layers
}

\author{
Yuling Luo ${ }^{1} \cdot$ Ronglong Zhou ${ }^{1} \cdot$ Junxiu Liu $^{1}$ (D) \\ Senhui Qiu ${ }^{1,2} \cdot \mathrm{Yi} \mathrm{Cao}^{3}$
}

Received: 17 April 2017 / Revised: 3 January 2018 / Accepted: 26 February 2018

(C) Springer Science+Business Media, LLC, part of Springer Nature 2018

\begin{abstract}
In this paper, we propose an efficient and self-adapting colour-image encryption algorithm based on chaos and the interactions among multiple red, green and blue (RGB) layers. Our study uses two chaotic systems and the interactions among the multiple layers to strengthen the cryptosystem for the colour-image encryption, which can achieve better confusion and diffusion performances. In the confusion process, we use the novel Rubik's Cube Scheme (RCS) to scramble the image. The significant advantage of this approach is that it sufficiently destroys the correlation among the different layers of colour image, which is the most important feature of the randomness for the encryption. The theoretical analysis and experimental results show that the proposed algorithm can improve the encoding efficiency, enhances the security of the cipher-text, has a large key space and high key sensitivity, and is also able to resist statistical and exhaustive attacks.
\end{abstract}

Keywords Colour-image encryption · Chaos $\cdot$ Interaction of multiple layers $\cdot$ Security analysis

\section{Introduction}

The security of multimedia data has become increasingly important due to the advances in networking technology coupled with the popularity of the internet [15]. Therefore, designing an effective and fast encryption method to ensure the safety of image data in a variety of communication network transmissions has received widespread attention [7]. However,

Junxiu Liu

liujunxiu@mailbox.gxnu.edu.cn

1 Guangxi Key Lab of Multi-Source Information Mining and Security, Faculty of Electronic Engineering, Guangxi Normal University, Guilin, China

2 Guangxi Experiment Centre of Information Science, Guilin, China

3 Department of Business Transformation and Sustainable Enterprise, Surrey Business School, University of Surrey, Surrey GU2 7XH, UK 
traditional image encryption schemes, e.g. Data Encryption Standard (DES) [32], Advanced Encryption Standard (AES) [10], and Rivest Shamir Adleman (RSA) et al. [6], (in which the image is represented as a plaintext for encryption), are not suitable for image encryption due to the special features of images, e.g. bulky data capacity, strong redundancy and high correlations between adjacent pixels [6]. Chaotic systems have many important properties, such as sensitive dependence on initial conditions and parameters, unpredictability, similar randomness, aperiodicity and ergodicity, which make chaotic systems popular in image encryption $[22,23]$. In the past 20 years, a large number of chaos-based encryption schemes have been designed $[12,16,50]$. According to the Shannon information theory, confusion and diffusion are two properties of the operation of a secure cipher in cryptography [27, 45, 46]. Specifically, confusion means that each binary digit/bit of the cipher-text should depend on several parts of the key, obscuring the connections between the two, and diffusion is that if a single bit of the plain-text is changed, then half of the bits in the cipher-text should be statistically changed, and similarly, if a one bit of the cipher-text is changed, then half of the bits in the plain-text should be statistically changed. Through much research and analysis [4, 8, 34], we have discovered that both confusion and diffusion are simultaneously employed in one image encryption scheme, resulting in substantial performance improvements.

However, by comparing the encryption performances of many confusion-diffusion schemes, it was found that most of them contain security defects [3, 26, 28]. For instance for the colour-image encryption schemes proposed in recent years [20, 25, 27, 33, 37, 41, 47], most of them used the same method to encrypt the red, green and blue layers [20]. They neglect the correlations among these layers and are more vulnerable to attacks. To overcome this problem, Wang et al. [36] presented a novel colour-image encryption algorithm based on the chaotic logistic map. The main advantage of this algorithm is the simultaneous encryption of the different layers of a colour image, taking into consideration their correlations. By using this algorithm, the correlation among the components is reduced, but the proposed algorithm also has some disadvantages: the key space is defined over periodic orbits of the chaotic map, which reduces the size of the space key; it uses data directly from the logistic map, which affects the encryption properties; and the security analyses of information entropy and time encryption are not included. The Wang algorithm [36] was efficiently cryptanalysed by a plainimage attack by Li et al. in the approach [18]. In addition in the approach [25], researchers proposed an RGB image encryption algorithm based on total plain-image characteristics and chaos, where the initial parameters of the chaotic system are gained through the plain-image pixel operations of summation and remainder. The advantages of this scheme are its simplicity and ease of design, but the disadvantage is obvious too. Using only the simple summation and remainder operations, it is difficult to reflect the main characteristics of the plain image. Luo et al. [23] used the quantification results for plain-image information and the external secret keys as the initial values of the chaotic system, to ensure that the initial parameters of the chaotic system are strict to plain image. High speed and plaintext sensitivity are the main advantages of this research, but the shortcoming is that the permutation result isn't ideal. This is because they construct the pseudo-random sequence using an unbalanced method, which leads to a poor randomness of the ergodic matrix. Then, Wang et al. proposed a new image encryption scheme [42], which used the simulated annealing algorithm to generate the random sequence for the confusion phase. The merit of this scheme is that the random sequence is generated excellently, but there is an obvious problem that some plain-image pixels still remain at their original positions after permutation. Several image encryption schemes based on cat map are presented [1, 6, 14, 17, 27, 48], such as 1-D cat map [1, 6, 27], 2-D cat map and 3-D 
cat map $[14,17,48]$. The main advantage of using the cat map is high computing speed. However, different types of cat maps suffer from some common problems. For example, a novel 3-D cat map-based spatial bit-level permutation strategy is proposed by Chen et al. [9]. This scheme cannot resist differential cryptanalysis, according to the analysis by Wang et al. [35]. In addition, the cat map is limited by the size of the plain image, the key space is also not large enough, and the secret key stays the same for different images [21]. Therefore, achieving self-adaption using the cat map is difficult which also lacks of universality.

According to the aforementioned analysis, it is clear that the previous approaches focused on improving the security and speed of the algorithm. Based on the comparison in the approaches [20, 25, 27, 33, 37, 41, 47], a good colour-image encryption scheme not only should encrypt the layers with different methods to reduce the correlations, but also the initial parameter of the chaotic system should be sensitive to plain images to resist plain-image attacks. The permutation algorithm should be designed reasonably, to achieve a good randomness performance $[6,14,17,23,42,48]$. In addition, according to the approaches [9, 36], a good algorithm should be universal for image encryption applications and have a large key space to resist differential cryptanalysis.

In this paper, we aim to design an efficient and self-adapting colour-image encryption algorithm based on chaos and interactions among multiple layers. We design a complex and effective quantification to improve the security of the cryptosystem. The multiple layers are inter-encrypted to reduce the correlations. Moreover, there are no shape or size limitations for the image. In addition, it can be made suitable for grey-image encryption with small changes. The main contributions of this paper are as follows. (1) A novel decimal quantification method based on the plain image is designed. (2) A novel permutation scheme, based on the Rubik's Cube Scheme (RCS), is constructed. (3) A new customized confusion-diffusion approach is designed. (4) The security analysis of the proposed image encryption scheme is presented in detail.

The rest of this paper is organized as follows. Section 2 describes the preliminary knowledge of the decimal quantification process and two chaotic maps. A novel RCS process is designed through the pixel cycle-shifts and image layer rotation. Section 3 presents the structure of the proposed cryptosystem, which includes the encryption and decryption processes. Section 4 analyses the security performance of the proposed cryptosystem. Section 5 gives a conclusion and discusses future work.

\section{Preliminaries}

In this section, we mainly introduce the decimal quantification process based on the plain image, two chaotic maps, and the process of the novel Rubik's Cube Scheme.

\subsection{The decimal quantification process}

In order to make the initial parameters be strict to plain-image information, we design a novel quantification method to obtain a decimal between $(0,1)$. Then, this decimal is used as the initial value or control parameter of the tent map to ensure that the generated chaotic sequence is strictly related to the pixel layers of the plain-image. Therefore, the performance of the image encryption can be improved significantly due to the close relationship between the plain image and the chaotic system. As shown in Fig. 1, the unidirectional quantification process consists of the following steps. 
Step 1. The grey value $\mathbf{I}(\mathrm{i}, \mathrm{j})$ of each pixel in the original image is firstly mapped to the range of $(0,360)$ in terms of the Eq. (1), which is denoted as $\mathbf{I 1}(i, j)$.

$$
\mathbf{I 1}(\mathrm{i}, \mathrm{j})=\mathbf{I}(\mathrm{i}, \mathrm{j}) \times 360 / 255
$$

Step 2. Transform the $\mathbf{I}(\mathrm{i}, \mathrm{j})$ to the real numbers $\mathbf{I 2}(\mathrm{i}, \mathrm{j})$ in the range of $(-1,1)$ in the accordance of the Eq. (2) by using the cosine function.

$$
\mathbf{I 2}(\mathrm{i}, \mathrm{j})=\cos (\mathbf{I 1}(\mathrm{i}, \mathrm{j}))
$$

Step 3. Set two parameters $\mathbf{p}$ and $\mathbf{q}$, and set their initial values are $\mathbf{p}=0$ and $\mathbf{q}=0$. Then calculate the values of $\mathbf{p}$ and $\mathbf{q}$ based on $\mathbf{I} 2(\mathrm{i}, \mathbf{j})$ in the terms of Eq. (3).

$$
\left\{\begin{array}{l}
\mathbf{p}=\mathbf{p}+\left(\frac{\mathbf{I} 2(\mathrm{i}, \mathrm{j})}{\pi} \times 10^{14} \bmod 1\right) ; \mathbf{I} 2(\mathrm{i}, \mathrm{j}) \geq 0 \\
\mathbf{q}=\mathbf{q}+\left(\frac{\mathbf{I} 2(\mathrm{i}, \mathrm{j})}{\pi} \times 10^{14} \bmod -1\right) ; \mathbf{I} 2(\mathrm{i}, \mathrm{j})<0
\end{array}\right.
$$

Step 4. Limit the values of $\mathbf{p}$ and $\mathbf{q}$ in the range of $(-1,1)$ by using the Eq. (4).

$$
\begin{cases}\mathbf{p}=\mathbf{p} \bmod 1 ; & \mathbf{p} \geq 1 \\ \mathbf{q}=\mathbf{q} \bmod -1 ; & \mathbf{q} \leq-1\end{cases}
$$

Step 5. Cycle the above process until the last pixel is processed, and get the last decimals $\mathbf{p}$ and $\mathbf{q}$.

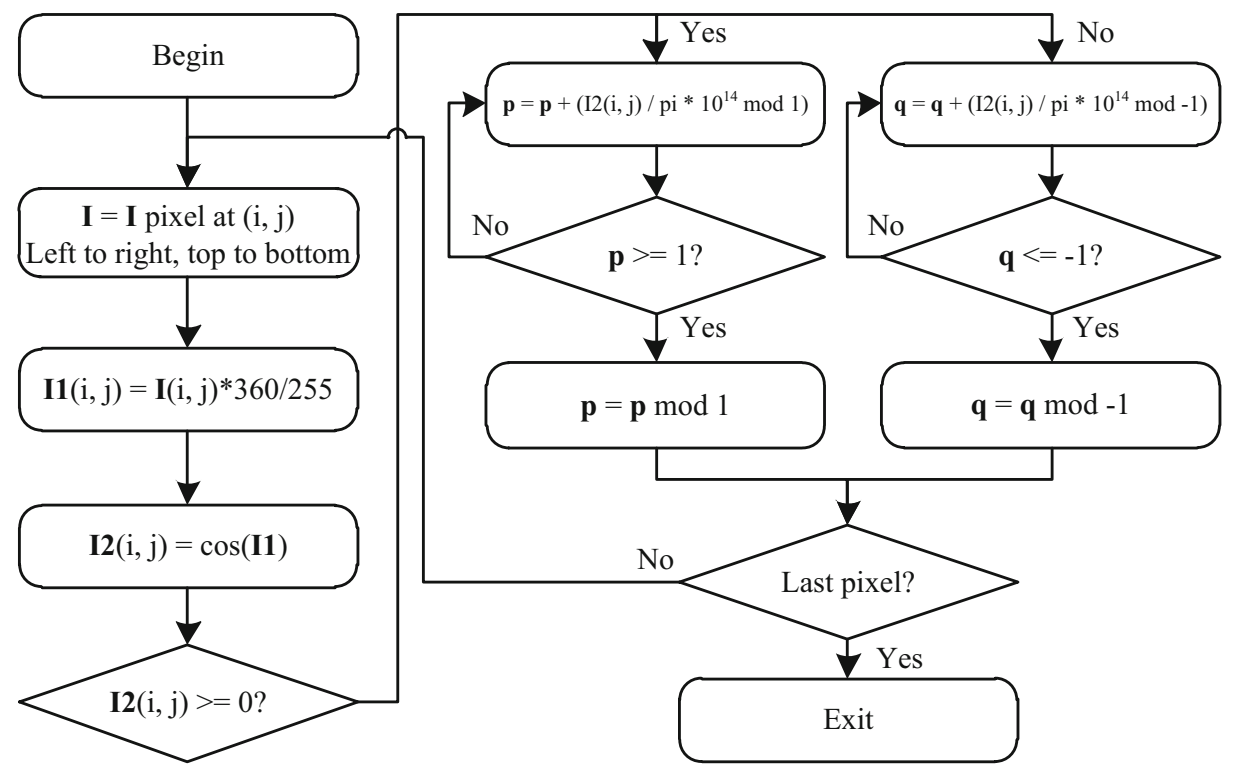

Fig. 1 The flow chart of the quantification process 
As shown above, especially in Step 3, it can be seen that the parameters of $\mathbf{p}$ and $\mathbf{q}$ are strictly related to the plain-image information, so it can enhance the performance of the image encryption. Specifically, the process described above can be separately applied to each of the three layers of the colour image. Therefore, we can further obtain three pairs of decimal values for $\mathbf{p}$ and $\mathbf{q}$. Then a decimal $\mathbf{k}$ can be obtained according to the Eq. (5), which will be applied to be the influence factor of the parameter $\eta$ of the PWLCM chaotic system in the following algorithm.

$$
\mathbf{k}=\left|\left(\mathbf{p}_{\mathbf{R}}-\mathbf{p}_{\mathbf{G}}+\mathbf{p}_{\mathbf{B}}\right)-\left(\mathbf{q}_{\mathbf{R}}-\mathbf{q}_{\mathbf{G}}+\mathbf{q}_{\mathbf{B}}\right)\right| \bmod 1
$$

Specifically, in order to make the control parameter $\eta$ of the PWLCM chaotic system in section 2.2 (b) be in the range of $(0,0.5)$, the $\mathbf{k}$ cannot equal to 0 because once be $\mathbf{k}$ is 0 , the control parameter $\eta$ of the PWLCM would be 0 in the terms of the following Eq. (14). Therefore, If the parameter $\mathbf{k}$ equals to 0 in the Eq. (5), the parameter $\mathbf{k}$ will be reset to be 0.19920307 , which makes the initial values and control parameters of the chaotic system unpredictable and improves the security of the encryption system effectively.

\subsection{The chaotic maps}

(a). The skew tent map is given by

$$
x(i)= \begin{cases}x(i-1) / a, & 0 \leq x(i-1)<a, \\ 1-x(i-1)) /(1-a), & a \leq x(i-1) \leq 1,\end{cases}
$$

where $a$ is the controlling parameter and $a \neq 0.5$. This map has high pseudo-randomness when $a$ is in the interval $(0,1)$. The skew tent map is widely used in cryptosystems due to the even distribution and high ergodicity [23].

To obtain chaotic sequences to improve the security of permutation for images, each state value of the tent map should be quantified to obtain ideal chaotic sequences by Eq. (7), where $w$ is an integer.

$$
x^{\prime}(i)=x(i) \times 10^{14} \bmod w+1, \quad i \in(1, w)
$$

(b). The PWLCM map is given by

$$
x(i+1)=F(x(i), \eta)= \begin{cases}\frac{x(i)}{\eta}, & 0<x_{i}<\eta \\ \frac{x(i)-\eta}{2-\eta}, & \eta \leq x_{i}<0.5 \\ F(1-x(i), \eta), & 0.5 \leq x_{i}<1\end{cases}
$$

where $x(i) \in(0,1)$. When control parameter $\eta \in(0,0.5)$, it evolves into a chaotic state, and $\eta$ can serve as a secret key. The PWLCM system has uniform invariant distribution and very good ergodicity, confusion and determinacy [31], thus it can provide an excellent random sequence that is suitable for the cryptosystem. 


\subsection{The process of the novel Rubik's Cube Scheme}

To illustrate this strategy properly, a colour image of size $\mathrm{M} \times \mathrm{N} \times 3$ (including the red, green and blue layers) is used for simplicity. Figure 2 is a diagram of the permutation process, and the detailed process is described below.

The cycle-shift and rotation operations for the red, green and blue pixel levels are different. The rows number of the image matrix at any processing stage is $L$. The scrambling process of the red layer of the image is as follows.

Step 1. Obtain three ergodic sequences in accordance with Eq. (6) and Eq. (7); they are denoted by $x_{R}^{\prime}(n), x_{G}^{\prime}(n)$ and $x_{B}^{\prime}(n)$. Specifically, the three initial values $x_{R}(1) x_{G}(1)$ and $x_{B}(1)$ of the skew tent map are produced by the characteristic values of the different colour layers of the plain image. For example, they can be obtained in two ways: 1) Calculate the percentage of " 1 " in each bit-plane matrix of plain-image. 2) Use the decimal quantification scheme in Section 2.1. And the number of iterations of the tent map is $w$ ( $w=$ $\max (\mathrm{M}, \mathrm{N}))$.

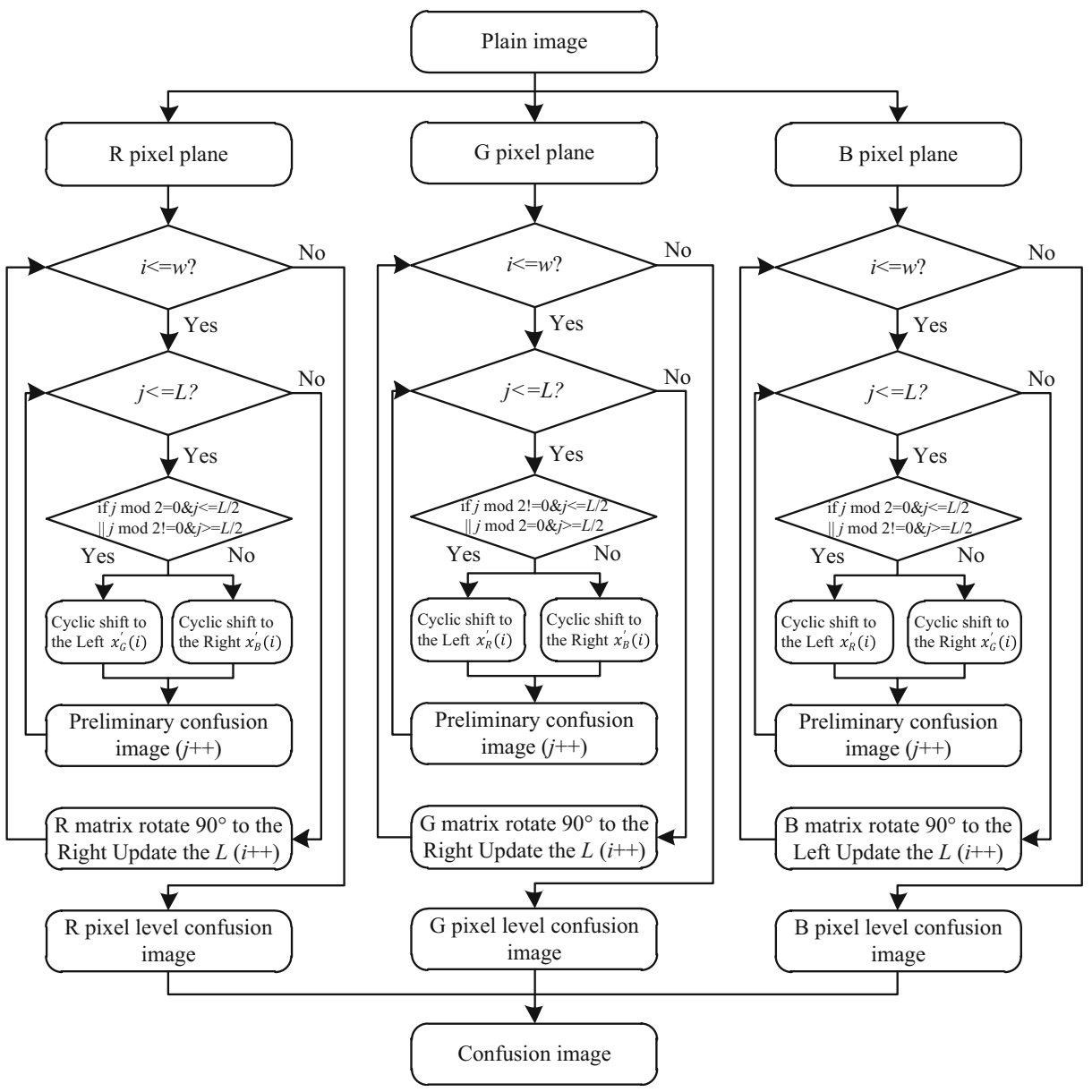

Fig. 2 The flowchart of the Rubik's cube permutation technology 
Step 2. Set $i=1$, and the $j$ th $\left(j \in e v e n \& j \leq \frac{L}{2}\right.$ or $\left.j \in o d d \& j \geq \frac{L}{2}\right)$ row of the red layer matrix is cycle-shifted to the left $x_{G}^{\prime}(i)$ times. Therefore, the other rows are cycle-shifted to the right $x_{B}^{\prime}(i)$ times, as shown in Eq. (9), where $i$ denotes the $i$ th integer of the sequence and $j$ denotes the $j$ th row of the R-layer matrix.

$$
\begin{cases}I_{R}(j,:, 1)=\operatorname{cycleft}\left(I_{R}(j,:, 1), x_{G}^{\prime}(i)\right) ; & \text { when } j \in e v e n \& j \leq \frac{L}{2} \text { or } j \in \text { odd } \& \geq \frac{L}{2} \\ I_{R}(j,:, 1)=\operatorname{cycright}\left(I_{R}(j,:, 1), x_{B}^{\prime}(i)\right) ; & \text { otherwise }\end{cases}
$$

Step 3. Rotate the generated red layer matrix by 90 degree to the right.

Step 4. Let $i=i+1$, and continue to cycle-shift and rotate the red layer matrix of the image according to Step 2 and Step 3.

Step 5. When $i>w$, the scrambling operation of the red layer is complete.

Similarly, the permutation processes of the green and blue layers of the image are almost the same, but there are some small differences.

(a). The direction of the cycle-shifted for the green layer is opposite that for the red layer. Specifically, the $j$ th $\left(j \in o d d \& j \leq \frac{L}{2}\right.$ or $\left.j \in e v e n \& j \geq \frac{L}{2}\right)$ row of the green layer matrix is cycle-shifted to the left $x_{R}^{\prime}(i)$ times, and the others are cycle-shifted to the right $x_{B}^{\prime}(i)$ times, as shown in Eq. (10).

$$
\left\{\begin{array}{l}
I_{G}(j,:, 2)=\operatorname{cycleft}\left(I_{G}(j,:, 2), x_{R}^{\prime}(i)\right) ; \quad \text { when } j \in \text { odd } \& j \leq \frac{L}{2} \text { or } j \in \text { even } \& \geq \frac{L}{2} \\
I_{G}(j,:, 2)=\text { cycright }\left(I_{G}(j,:, 2), x_{B}^{\prime}(i)\right) ; \text { otherwise }
\end{array}\right.
$$

(b). The direction of the rotation angle for the blue layer is opposite that for the red layer. Specifically, in Step 2, the new matrix is rotated by 90 degree to the left, and the shiftcontrolling parameters are $x_{R}^{\prime}(i)$ and $x_{G}^{\prime}(i)$. The specific process is shown in Eq. (11).

$$
\left\{\begin{array}{l}
I_{B}(j,:, 3)=\operatorname{cycleft}\left(I_{B}(j,:, 3), x_{R}^{\prime}(i)\right) ; \quad \text { when } j \in \text { even } \& j \leq \frac{L}{2} \text { or } j \in \text { odd } \& \geq \frac{L}{2} \\
I_{B}(j,:, 3)=\operatorname{cycright}\left(I_{B}(j,:, 3), x_{G}^{\prime}(i)\right) ; \text { otherwise }
\end{array}\right.
$$

\section{The proposed image-encryption}

In this section, we present the encryption and decryption processes in detail. The encryption process includes two parts: the confusion process and the diffusion process. A flow chart of the confusion and diffusion processes is shown in Fig. 3. In the confusion process, we utilize the proposed quantification and RCS methods to permute the plain image. In the diffusion process, the image is converted to the bit-plane, and the three percentage values, represented as initial decimals of the PWLCM map, are obtained from the three bit-planes of the colour image. Then, the three corresponding chaotic sequences are calculated to determine the interaction and inter-diffusion of the three layers. Finally, the encrypted image is obtained. 


\subsection{The process of image encryption}

A good encryption scheme should have the following features: (a) It should act directly on an image of any size for the image encryption application, rather than dividing the image into blocks of the same size [25]. (b) It should have a key stream that is strongly related to the plain image. When we encrypt different plain images, the encryption scheme should generate different key streams. The proposed method satisfies these properties, and the size of the colour plain image is assumed to be $\mathrm{M} \times \mathrm{N}$. The encryption process is given as follows.

Step 1. The three image layers are extracted using the quantification method proposed in section 2.1, and then the decimal $\mathbf{k}$ is obtained as the control parameter $a$ of the skew tent map.

Step 2. According to the same quantification method, three new groups of decimals, $\left(\mathbf{p}_{\mathbf{R}}\right.$, $\left.\mathbf{q}_{\mathbf{R}}\right),\left(\mathbf{p}_{\mathbf{G}}, \mathbf{q}_{\mathbf{G}}\right)$ and $\left(\mathbf{p}_{\mathbf{B}}, \mathbf{q}_{\mathbf{B}}\right)$, are obtained, in the order from bottom to top and right to left. Then, the three decimals $x_{R}(1), x_{G}(1)$ and $x_{B}(1)$ are generated by Eq. (12), which correspond to the three layers, respectively. Three common image size 256, 512 and 1024 are introduced to be the second parameters in Eq. (12) in order to assure the executive efficiency of the encryption system. $x_{R}(1), x_{G}(1)$ and $x_{B}(1)$ are used as initial values of the skew tent map.

$$
\left\{\begin{array}{l}
x_{R}(1)=\left(\left(1+\mathbf{q}_{\mathbf{R}}\right) \times \mathbf{p}_{\mathbf{R}}+\left|\frac{\bmod \left(\sum_{\mathrm{i}=1}^{\mathrm{M}} \sum_{\mathrm{j}=1}^{\mathrm{N}} \mathrm{I}_{\mathrm{R}}(\mathrm{i}, \mathrm{j}), 256\right)}{256} \times \mathbf{q}_{\mathbf{R}}\right|\right) ; \\
x_{G}(1)=\left(\left(1+\mathbf{q}_{\mathbf{G}}\right) \times \mathbf{p}_{\mathbf{G}}+\left|\frac{\bmod \left(\sum_{\mathrm{i}=1}^{\mathrm{M}} \sum_{\mathrm{j}=1}^{\mathrm{N}} \mathrm{I}_{\mathrm{G}}(\mathrm{i}, \mathrm{j}), 512\right)}{512} \times \mathbf{q}_{\mathbf{G}}\right|\right) ; \\
x_{B}(1)=\left(\left(1+\mathbf{q}_{\mathbf{B}}\right) \times \mathbf{p}_{\mathbf{B}}+\left|\frac{\bmod \left(\sum_{\mathrm{i}=1}^{\mathrm{M}} \sum_{\mathrm{j}=1}^{\mathrm{N}} \mathrm{I}_{\mathrm{B}}(\mathrm{i}, \mathrm{j}), 1024\right)}{1024} \times \mathbf{q}_{\mathbf{G}}\right|\right) .
\end{array}\right.
$$

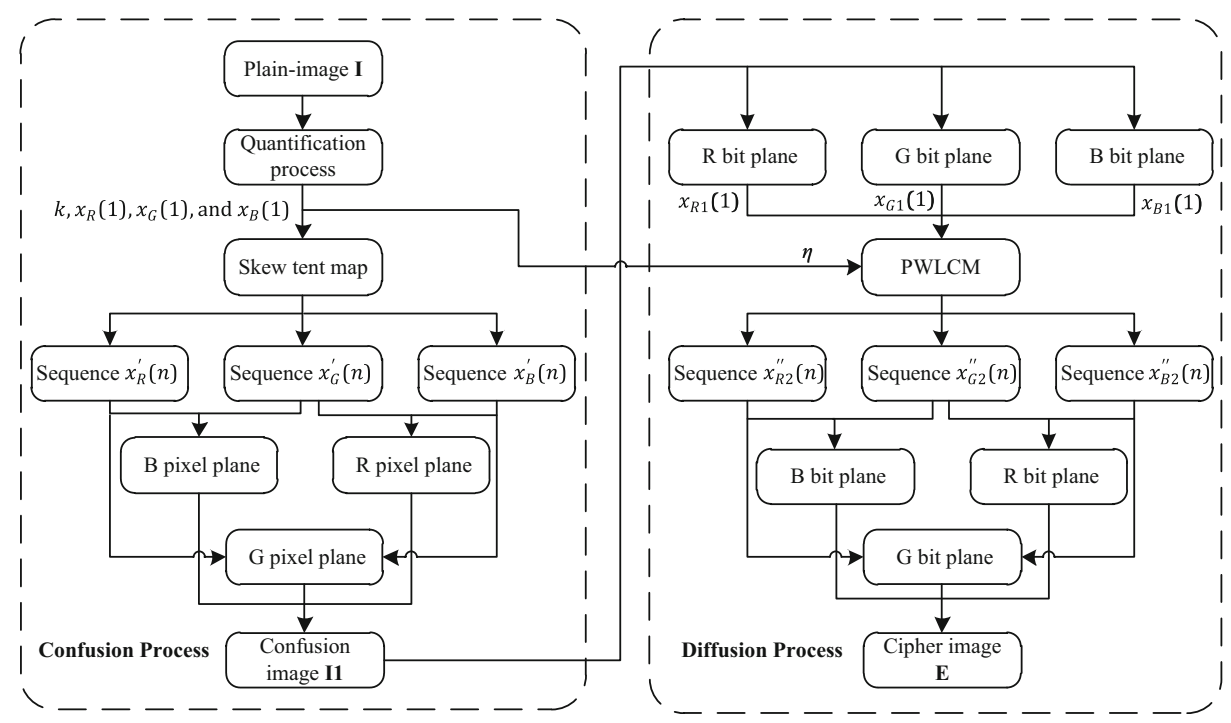

Fig. 3 The block diagram of the proposed encryption scheme 
Step 3. The skew map is iterated $w(w=\max (\mathrm{M}, \mathrm{N}))$ times to produce three sequences $x_{R}(n)$, $x_{G}(n)$ and $x_{B}(n)$.

Step 4. The chaotic sequences calculated in Step 3 are quantified by Eq. (13), and then three new sequences $x_{R}^{\prime}(n), x_{G}^{\prime}(n)$ and $x_{B}^{\prime}(n)$ are obtained.

$$
x_{\text {RorGorB }}^{\prime}(i)=x_{\text {RorGorB }}(i) \times 10^{14} \bmod w+1 \quad \mathrm{i} \in(1, w)
$$

Step 5. The Rubik's Cube Scheme (RCS) from section 2.3 is applied to obtain the scrambled image I1.

Step 6. Transform the image I1 to bit-plane from the pixel plane, and get three binary matrices (R, G and B). And the percentage of " 1 " in each bit-plane matrix of $\mathbf{I 1}$ is calculated, and then three decimals are obtained, which are used for the initial values $x_{R 1}(1)$, $x_{G 1}(1)$ and $x_{B 1}(1)$ of the PWLCM system. Then the value $\eta$ is calculated by using Eq. (14) as the control parameter of the PWLCM map where $\mathbf{k}$ comes from Step 1.

$$
\eta=\left(0.9 \times \mathbf{k}+\frac{\bmod \left(\sum_{\mathrm{i}=1}^{\mathrm{M}} \sum_{\mathrm{j}=1}^{\mathrm{N}} \mathrm{I}(\mathrm{i}, \mathrm{j}), 256\right)}{256} \times 0.1\right) / 2
$$

Step 7. The PWLCM map is iterated $\mathrm{M} \times \mathrm{N}$ times and the three chaotic sequences $x_{R 2}(n)$, $x_{G 2}(n)$ and $x_{B 2}(n)$ are obtained. After quantifying them using Eq. (15), they are converted into binary arrays. The size of each array is $\mathrm{L}(\mathrm{L}=\mathrm{M} \times \mathrm{N} \times 8)$. Here, RorGorB represents that the same operation is performed for any layer of the color image, and the role of $[x]_{2}$ is to convert pixel value $x$ to binary data.

$$
x_{\text {RorGorB }}^{\prime \prime}(i)=\left[x_{R 2 \operatorname{orG} 2 o r B 2} \times 10^{14} \bmod 256+1\right]_{2}, i \in(1, \mathrm{M} \times \mathrm{N})
$$

Step 8. The three RGB bit-plane matrixes are converted to one-dimensional row vectors $x_{R 2}^{\prime \prime}(n), x_{G 2}^{\prime \prime}(n)$ and $x_{B 2}^{\prime \prime}(n)$. Then, the three RGB row vectors are inter-encrypted as follows.

The sequences $x_{G}^{\prime}(n)$ and $x_{B}^{\prime}(n)$ are used as control parameters for the red layer XOR operations. The first entry of the red layer row vector is encrypted by Eq. (16), and then the $i$ th entry is encrypted as shown in Eq. (17).

$$
\begin{gathered}
\mathbf{E} x_{R 2}^{\prime \prime}(1)=x_{R 2}^{\prime \prime}(1) \oplus x_{R 2}^{\prime \prime}(\mathrm{L}) \oplus x_{G}^{\prime \prime}(1) \oplus x_{B}^{\prime \prime}(1) \\
\mathbf{E} x_{R 2}^{\prime \prime}(i)=x_{R 2}^{\prime \prime}(i) \oplus x_{R 2}^{\prime \prime}(\mathrm{L}-i) \oplus x_{G}^{\prime \prime}(i) \oplus x_{B}^{\prime \prime}(i) \quad(2 \leq i \leq \mathrm{L})
\end{gathered}
$$

The green and blue layer encryptions are similar to that of the red layer, with different parameters. The bit-XOR operations are shown in Eq. (18-19) and Eq. (20-21), respectively.

$$
\begin{gathered}
\mathbf{E} x_{G 2}^{\prime \prime}(1)=x_{G 2}^{\prime \prime}(1) \oplus x_{G 2}^{\prime \prime}(\mathrm{L}) \oplus x_{R}^{\prime \prime}(1) \oplus x_{B}^{\prime \prime}(1) \\
\mathbf{E} x_{G 2}^{\prime \prime}(i)=x_{G 2}^{\prime \prime}(1) \oplus x_{G 2}^{\prime \prime}(\mathrm{L}-i) \oplus x_{R}^{\prime \prime}(i) \oplus x_{B}^{\prime \prime}(i)(2 \leq i \leq \mathrm{L})
\end{gathered}
$$




$$
\begin{gathered}
\mathbf{E} x_{B 2}^{\prime \prime}(1)=x_{B 2}^{\prime \prime}(1) \oplus x_{B 2}^{\prime \prime}(\mathrm{L}) \oplus x_{R}^{\prime \prime}(1) \oplus x_{G}^{\prime \prime}(1) \\
\mathbf{E} x_{B 2}^{\prime \prime}(i)=x_{B 2}^{\prime \prime}(i) \oplus x_{B 2}^{\prime \prime}(\mathrm{L}-i) \oplus x_{R}^{\prime \prime}(i) \oplus x_{G}^{\prime \prime}(i) \quad(2 \leq i \leq \mathrm{L})
\end{gathered}
$$

Step 9. Finally, we obtain three binary sequences. The image of size $\mathrm{M} \times \mathrm{N}$ is recovered in the pixel-plane, and the encrypted colour image $\mathbf{E}$ is finally obtained.

After these steps, the plain image is completely encrypted. In the decryption process, these steps are applied in reverse order which is given in next subsection.

\subsection{The process of image decryption}

Step 1. First, the three sequences $x_{R}^{\prime \prime}(n), x_{G}^{\prime \prime}(n)$ and $x_{B}^{\prime \prime}(n)$ are obtained according to the Step 6 and Step 7 in section 3.1.

Step 2. The cipher image is processed by one round of bit-XOR operations, as in the method described in Step 8 of section 3.1 but in reverse order.

Step 3. Then, we get three chaotic integer sequences $x_{R}^{\prime}(n), x_{G}^{\prime}(n)$ and $x_{B}^{\prime}(n)$ according to Step 1-Step 5 in section 3.1.

Similarly, the number of rows of the processed image at any stage is $L$. The decryption process of the red layer of the image is given by:

(a). Set $i=w$. The $j$ th $\left(j \in e v e n \& j \leq \frac{L}{2}\right.$ or $\left.j \in o d d \& j \geq \frac{L}{2}\right)$ row of the red layer matrix is cycleshifted to the right $x_{G}^{\prime}(i)$ times, so the other rows are cycle-shifted to the left $x_{B}^{\prime}(i)$ times.

(b). After the cycle-shift, the generated matrix for the red layer is rotated 90 degree to the left.

(c). Let $i=i-1$, and then repeat (a) and (b).

(d). When $i<1$, the decryption operation of the red layer is complete.

The direction of cycle-shifted for the green layer is opposite that of the red layer, and the controlling parameters are $x_{R}^{\prime}(i)$ and $x_{B}^{\prime}(i)$, respectively. The direction of the rotation angle for the blue layer is opposite that for the red layer, and the controlling parameters are $x_{R}^{\prime}(i)$ and $x_{G}^{\prime}(i)$, respectively. Then, through confusion and diffusion in the corresponding reverse order, we obtain the decrypted image.

\section{Experimental results}

To test the performance of the proposed algorithm, the experiments are carried out and the results are given in this section. We use the three standard $512 \times 512$ colour images shown in Fig. 4 (a-c) as the input images (the images are "jpg" format). The experiment is run in MATLAB 2014a. Then, the permuted images are shown in Fig. 4 (d-f), the encrypted images are shown in Fig. 4 (g-i), and the decrypted images are shown in Fig. 4 (j-1). Of course, the experimental results show that the algorithm is also applicable to other image formats such as "png", "bmp" and "tiff". A good encryption algorithm should resist all known kinds of attacks, such as exhaustive attacks, differential attacks and statistical attacks. In addition, the key space 
should be large enough to resist brute-force attacks and should be sensitive to the secret keys. Therefore, in the following subsections, several tests are discussed to check the security of the proposed cryptosystem.

\subsection{Key space analysis}

High sensitivity to initial conditions is an inherent characteristic of any chaotic system. To provide an encryption algorithm with high security, the key space should be large enough to make any brute-force attack ineffective. There are two chaotic systems contained in the proposed cryptosystem: the skew tent map and PWLCM map. The skew tent map contains a control parameter $a$ and initial parameters $x_{R}(1), x_{G}(1)$, and $x_{B}(1)$, and the PWLCM map contains these parameters $\eta$, $x_{R 1}(1), x_{G 1}(1)$ and $x_{B 1}(1)$. If the precision is $10^{-15}$, the key space will be $\left(10^{15}\right)^{8} \approx 2^{400}$. In
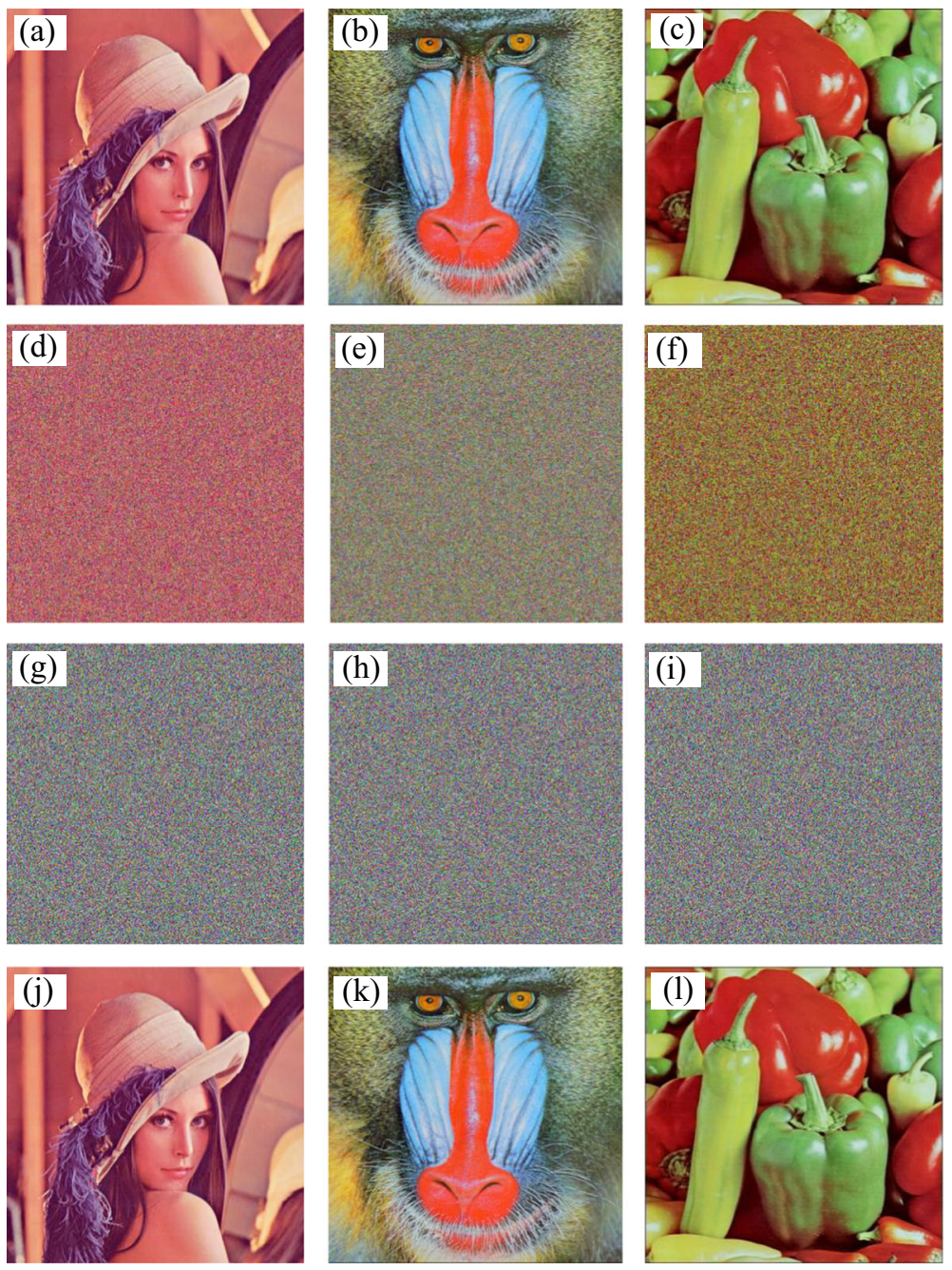

Fig. 4 Original, permuted, encrypted and decrypted images: (a), (d), (g), (j) Lena; (b), (e), (h), (k) Baboon; (c), (f), (i) (1) Peppers 
addition, these parameters are be strictly related with the plain image, so the key space is much larger than $2^{400}$, which is large enough to prevent exhaustive searching. Thus, the proposed algorithm is fully capable of resisting any brute-force attack.

\subsection{Key-sensitivity analysis}

A good encryption algorithm should be sensitive to the secret key. In other words, a very small change in the secret key will result in a huge change in the encrypted image. In the proposed algorithm, we calculated the tent map initial control parameter $a=0.412734985351563$ to encrypt the $512 \times 512$ Lena colour image in section 2.1 . We change the parameter to $a=$ 0.412734985351564 , which is just one bit different from the initial value, and keep other keys unchanged. The images encrypted with the correct and incorrect keys are shown in Fig. 5 (a)
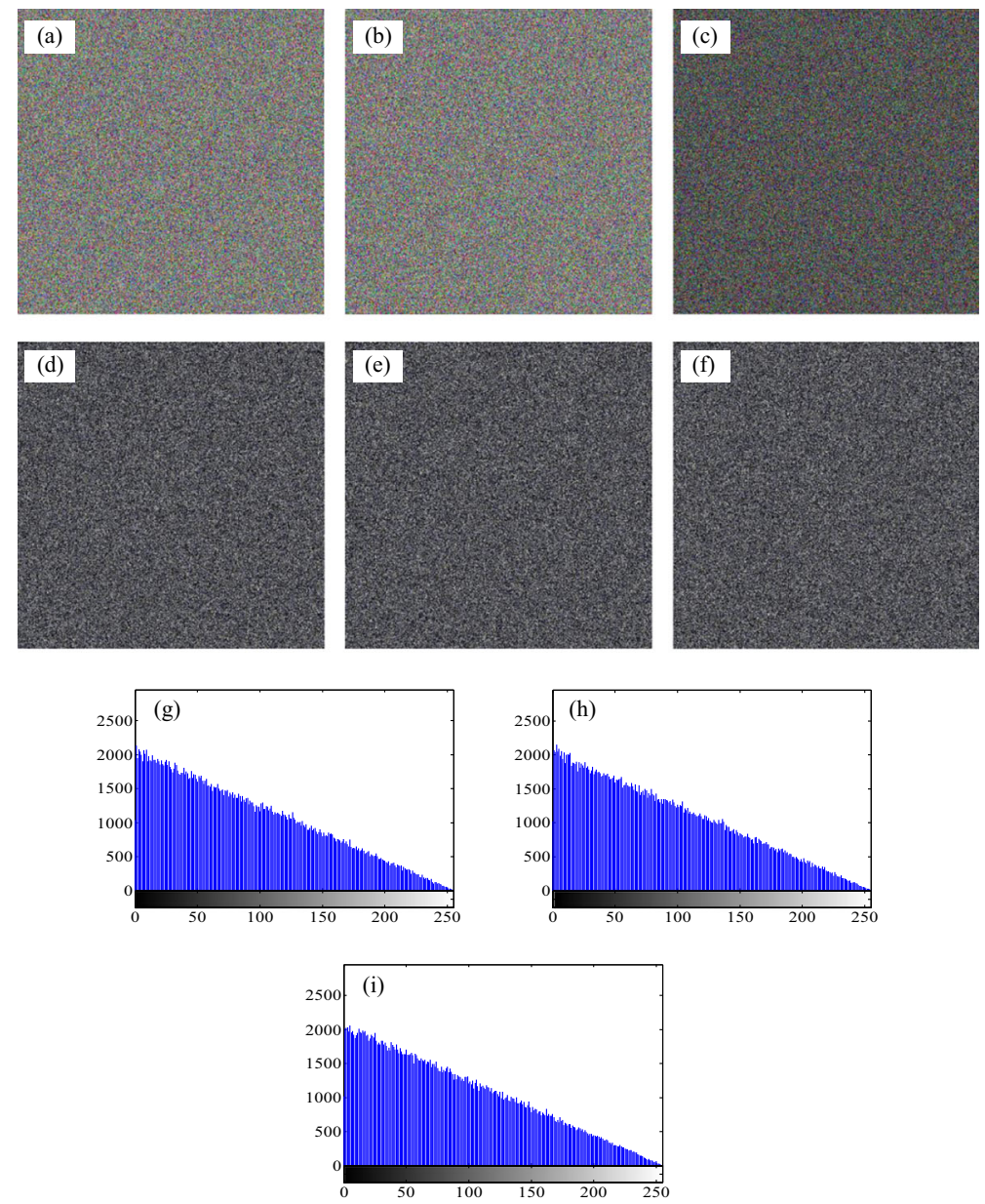

Fig. 5 The key-sensitivity results. (a) is original cipher image; (b) is cipher image with a slight change in the initial key; (c) shows the differences between the original cipher image (a) and image (b); (d) is the red layer of image (c); (e) is the green layer of image (c); (f) is the blue layer of image (c); (g)-(i) are the grey histograms of image (d)-(f), respectively 
and Fig. 5 (b), respectively. The difference between the two encrypted images is shown in Fig. 5 (c), and the corresponding NPCR and UACI values are $99.63 \%$ and $33.54 \%$, respectively. Figure 5 (d), (e) and (f) are red, green and blue layers of Fig. 5 (c), respectively. Fig. 5 (g), (h) and (i) are the histogram of Fig. 5 (d), (e) and (f), respectively. Similar sensitivity results are obtained when we change the other parameters by only one bit. This clearly demonstrates that the proposed cryptosystem is highly sensitive to the secret key.

\subsection{Differential attack}

Attackers often make small changes to the original image and then encrypt the image using the proposed algorithm to determine the relationship between the original image and the cipher image. Therefore, the cryptosystem should be sensitive to the plain image to resist the differential attack. Some quantitative measures are the number of pixels change rate (NPCR) and the unified average changing intensity (UACI). These are used to test the influence of a small change on the whole cipher image. $\mathrm{NPCR}_{R, G, B}$ and $\mathrm{UACI}_{R, G, B}$ are defined as follows.

$$
\mathrm{NPCR}_{R, G, B}=\frac{\sum_{i, j} D_{R, G, B}(i, j)}{M \times N} \times 100 \%,
$$

where $\mathrm{M}$ and $\mathrm{N}$ are the width and height of the cipher image, $E_{R, G, B}$ and $E_{R, G, B}^{\prime}$ are the two cipher images whose corresponding plain images differ by only one bit. The difference array $D_{R, G, B}(i, j)$ is determined by the following rule: when the pixels in position $(i, j)$ in $E_{R, G, B}$ and $E_{R, G, B}^{\prime}(i, j)$ are different (i. e. $\left.E_{R, G, B} \neq E_{R, G, B}^{\prime}(i, j)\right)$, then $D_{R, G, B}(i, j)=1$; otherwise, $D_{R, G, B}(i$, $j$ ) is 0 . The probability distribution of $D_{R, G, B}(i, j)$ is

$$
D_{R, G, B}(i, j)=\left\{\begin{array}{l}
0, \text { if } E_{R, G, B}(i, j)=E_{R, G, B}^{\prime}(i, j), \\
1, \text { if } E_{R, G, B}(i, j) \neq E_{R, G, B}^{\prime}(i, j) .
\end{array}\right.
$$

In this test, the size of the colour image is $256 \times 256$, the quantitative measures NPCR and UACI are calculated for three different plain images as shown in Fig. 4 (a), (b) and (c), respectively, and their results are shown in Table 1 . The tested pixels are located at $(1,1)$ in each image.

From Table 1, the average values of NPCR and UACI are $99.6262 \%$ and $33.5586 \%$, respectively. 100 pixels from the red layer of the "Lena" image $(256 \times 256)$ are randomly selected, and each pixel's lowest bit is changed successively to obtain 100 different images. Then, we calculate the values of NPCR and UACI as shown in Fig. 6. We clearly find that the means of these 100 tests for NPCR and UACI are $99.6235 \%$ and $33.5378 \%$, respectively. Moreover, the different encryption algorithms are compared and the NPCR and UACI values

Table 1 NPCR and UACI for different plain images

\begin{tabular}{llllllll}
\hline Image & \multicolumn{3}{l}{ NPCR $(\%)$} & & & \multicolumn{2}{l}{ UACI $(\%)$} \\
\cline { 2 - 4 } & Red & Green & Blue & & Red & Green & Blue \\
\hline Lena & 99.6201 & 99.6445 & 99.6140 & 33.5903 & 33.5229 & 33.6609 \\
Baboon & 99.6490 & 99.6154 & 99.6017 & 33.5236 & 33.5441 & 33.5511 \\
Peppers & 99.6231 & 99.6221 & 99.6419 & 33.5112 & 33.5597 & 33.5634 \\
\hline
\end{tabular}



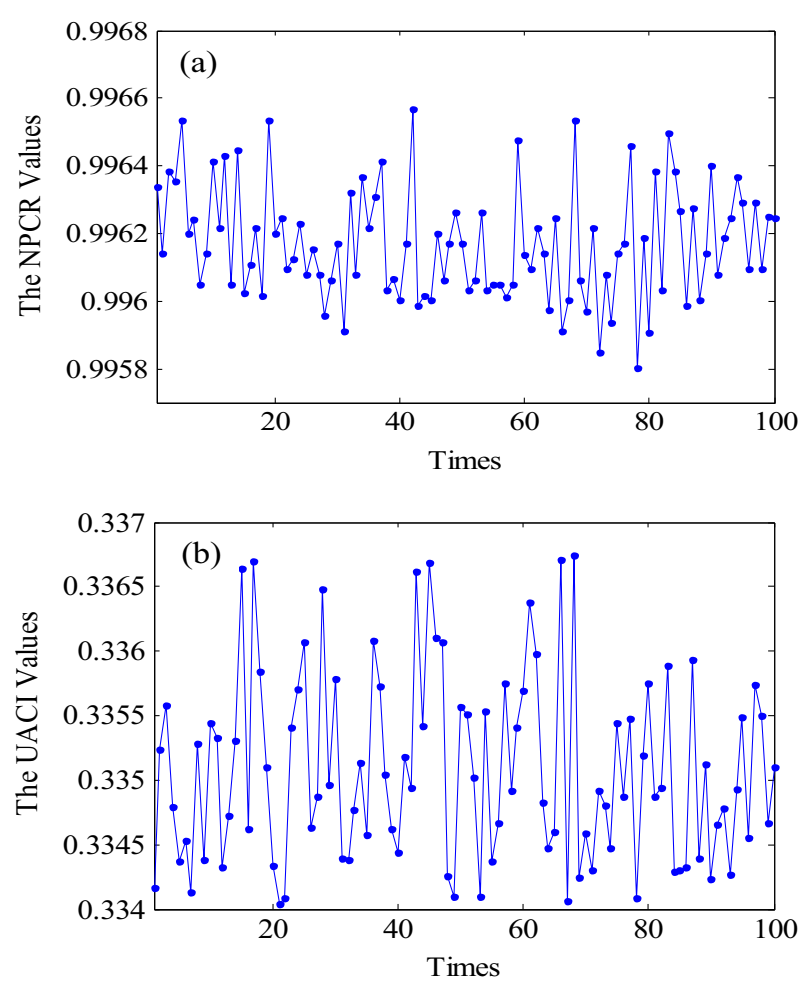

Fig. 6 The results of (a) NPCR and (b) UACI for all modified plain images using the Lena as an example

are shown in Table 2. From Table 2, we can see that the proposed algorithm is capable of resisting differential attacks.

Besides, if there is no the quantization process, the parameter $\mathbf{k}=0.2$ is fixed for the encryption algorithm to experiment and test. Similarly, the 100 pixels of the plain-image Lena are randomly changed to test the differential attack, i.e., the numbers of NPCR for these 100 times of experiments without the quantization process but only a fixed $\mathbf{k}$ are obtained, and they are shown in Fig. 7 to compare with the results above. The results show that the proposed quantization process can effectively enhance the sensitivity of the encryption algorithm to plain image.

In addition, in order to further test this effect of the quantization process, other plain images Baboon and Peppers are also experimented the same test. The experimental results are shown in Figs. 8 and 9, respectively. From the figures it can be clearly seen that there is a good performance as well. Therefore, these results illustrate that the quantization process can improve the security of encryption algorithm to certain extent.

Table 2 Comparative study of NPCR and UACI of the proposed algorithm $v s$. existing algorithms

\begin{tabular}{llllllll}
\hline Parameters & Ours & Ref. [23] & Ref. [36] & Ref. [42] & Ref. [43] & Ref. [5] & Ref. [29] \\
\hline NPCR (\%) & 99.6235 & 99.6109 & 99.6475 & 99.6000 & 99.6300 & 99.6167 & 99.3700 \\
UACI (\%) & 33.5378 & 33.4739 & 33.3343 & 33.4800 & 33.3700 & 33.4500 & 33.4500 \\
\hline
\end{tabular}




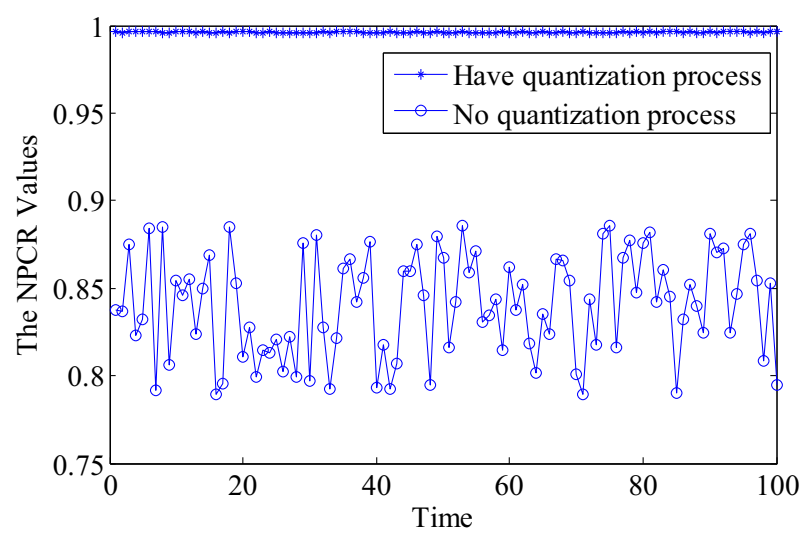

Fig. 7 The comparative NPCR values of the differential attack for plain image "Lena" with the quantization process and without the quantization process

\subsection{Information entropy}

Information entropy can be used to describe the complexity and randomness of the encryption system. Let $m$ be the information source, the information entropy can be calculated by:

$$
H(m)=\sum_{i=0}^{2^{n}-1} p\left(m_{i}\right) \log _{2} \frac{1}{p\left(m_{i}\right)}
$$

where $p\left(m_{i}\right)$ represents the probability of symbol $m_{i}$. For a random image with 256 grey levels, assume any grey level appears with the same probability. According to Eq. (25), we can achieve the ideal $H(m)=8$, which shows that the information is random. The closer the information entropy of the cipher image is to 8 , the higher the randomness of the cryptosystem. We calculate the information entropies of the cipher images in Fig. 4 (g), (h), and (i). The results are shown in Table 3. The entropy values are all very close to the ideal value of 8 , and the proposed scheme performs better than the other schemes.

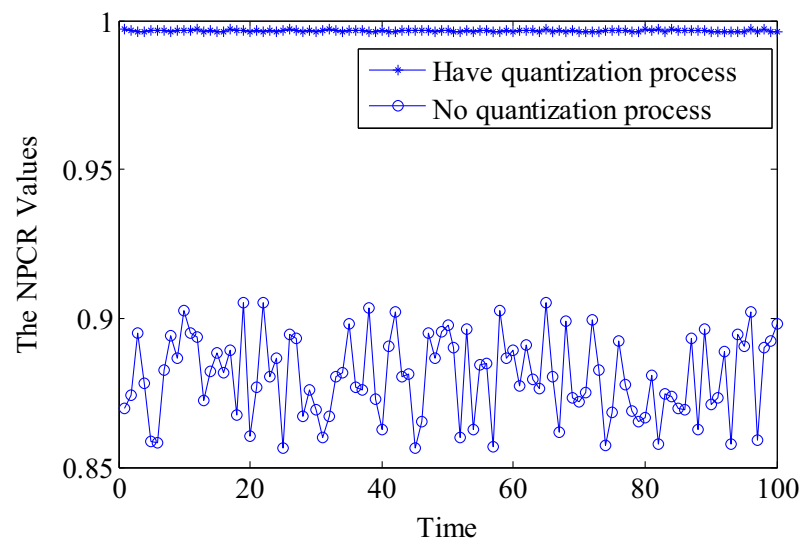

Fig. 8 The comparative NPCR values of the differential attack for plain image "Baboon" with the quantization process and without the quantization process 


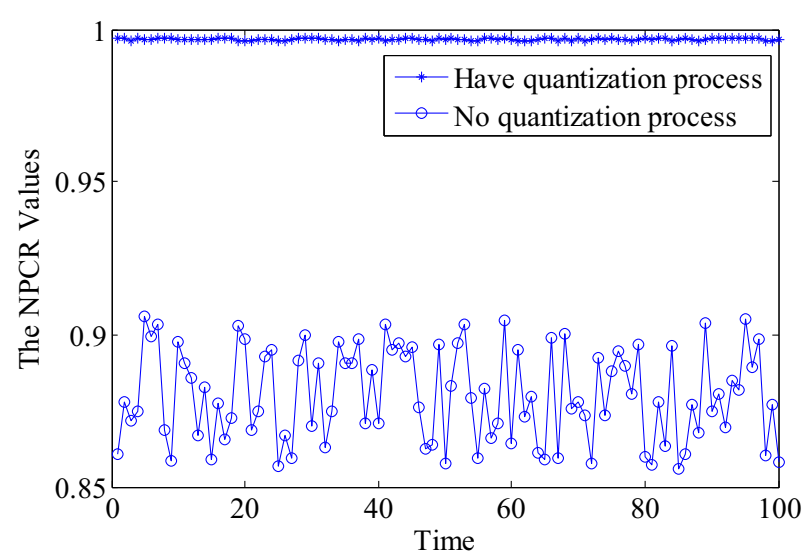

Fig. 9 The comparative NPCR values of the differential attack for plain image "Peppers" with the quantization process and without the quantization process

\subsection{Statistical analysis}

\subsubsection{The histogram analysis}

The histogram of an image reflects the contribution of each grey level. In general, the distribution of an ordinary plain image is nonuniform, and some values have high frequencies. Hence, it can be exploited easily due to the amount of useful information exposed. To resist such attacks, the histogram of the encrypted image should be uniform. The histograms of the plain image Fig. 4 (a) and cipher image Fig. 4 (d) are shown in Fig. 10. Comparing the histograms before and after encryption, we clearly find that the histogram of the encrypted image is very uniform and significantly different from that of the corresponding plain image.

\subsubsection{Correlation-coefficient analysis}

A plain image usually contains a lot of information due to the strong correlations between adjacent pixels. In general, the high correlations among adjacent pixels for an ordinary image exist in the horizontal, vertical and diagonal directions. Therefore, a good encryption algorithm should remove these correlations as soon as possible. The correlation property can be quantified by means of correlation coefficients as

Table 3 Comparative study of the entropy of the proposed algorithm vs. some existing algorithms

\begin{tabular}{lc}
\hline Algorithms & $H(m)$ \\
\hline This work (Lena) & 7.9998 \\
This work (Baboon) & 7.9997 \\
This work (Peppers) & 7.9998 \\
Ref. [15] & 7.9992 \\
Ref. [49] & 7.9993 \\
Ref. [20] & 7.9993 \\
Ref. [43] & 7.9970 \\
Ref. [5] & 7.9977 \\
Ref. [29] & 7.9920 \\
\hline
\end{tabular}



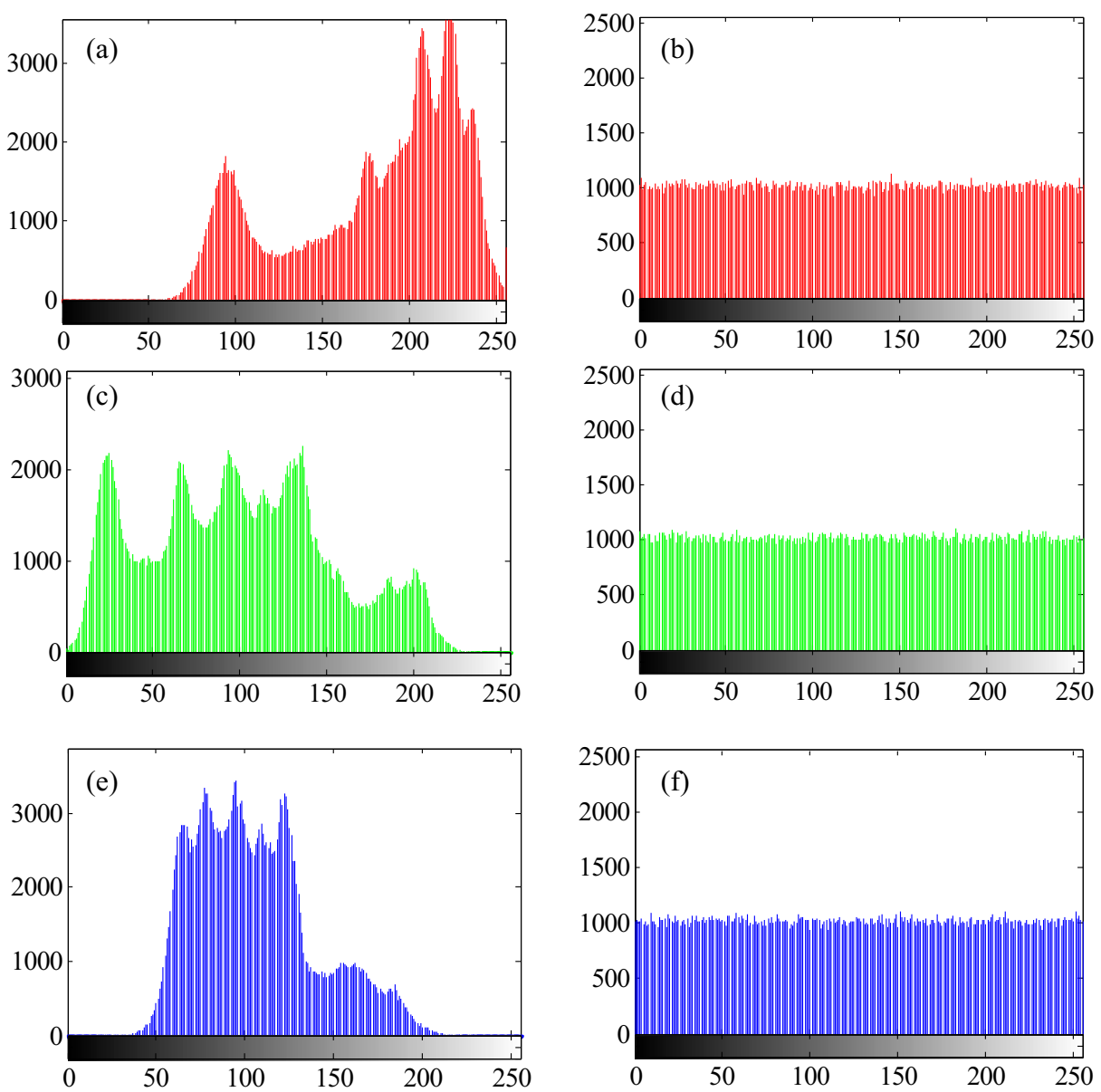

Fig. 10 (a)-(c) the histograms of initial colour image "Lena" for the red, green and blue layers, respectively. (d)(f) the histograms of the cipher image for the red, green and blue layers, respectively

$$
\mathrm{r}_{\mathrm{xy}}=\frac{\operatorname{cov}(\mathrm{x}, \mathrm{y})}{\sqrt{\mathrm{D}(\mathrm{x}) \mathrm{D}(\mathrm{y})}}
$$

where $\mathrm{x}$ and $\mathrm{y}$ are grey scale values of two adjacent pixels, and

$$
\begin{gathered}
\mathrm{E}(\mathrm{x})=\frac{1}{\mathrm{~N}} \sum_{\mathrm{i}=1}^{\mathrm{N}} \mathrm{x}_{\mathrm{i}} \\
\mathrm{D}(\mathrm{x})=\frac{1}{\mathrm{~N}} \sum_{\mathrm{i}=1}^{\mathrm{N}}\left(\mathrm{x}_{\mathrm{i}}-\mathrm{E}(\mathrm{x})\right)^{2} \\
\operatorname{cov}(\mathrm{x}, \mathrm{y})=\frac{1}{\mathrm{~N}} \sum_{\mathrm{i}=1}^{\mathrm{N}}\left(\mathrm{x}_{\mathrm{i}}-\mathrm{E}(\mathrm{x})\right)\left(\mathrm{y}_{\mathrm{i}}-\mathrm{E}(\mathrm{y})\right)
\end{gathered}
$$

where $\mathrm{x}_{\mathrm{i}}$ and $\mathrm{y}_{\mathrm{i}}$ are two adjacent pixels in the image, $\mathrm{N}$ is the total number of adjacent pixel pairs $\left(\mathrm{x}_{\mathrm{i}}, \mathrm{y}_{\mathrm{i}}\right)$, and $\mathrm{E}(\mathrm{x})$ and $\mathrm{E}(\mathrm{y})$ are the mean values of $\mathrm{x}_{\mathrm{i}}$ and $\mathrm{y}_{\mathrm{i}}$, respectively. First, we randomly select $10^{4}$ pairs adjacent pixels of "Lena" in the vertical, horizontal and diagonal 
directions from the plain and cipher images. The results as shown in Fig. 11. It is obvious that the plain image has a strong correlation among adjacent pixels. However, the the correlation in corresponding the cipher image becomes less relevant.

Then, the correlation coefficients of all three plain-images and their corresponding cipher images in vertical, horizontal and diagonal directions are calculated and shown in Table 4. It is clear that all correlation coefficients of the plain image are close to 1 ,
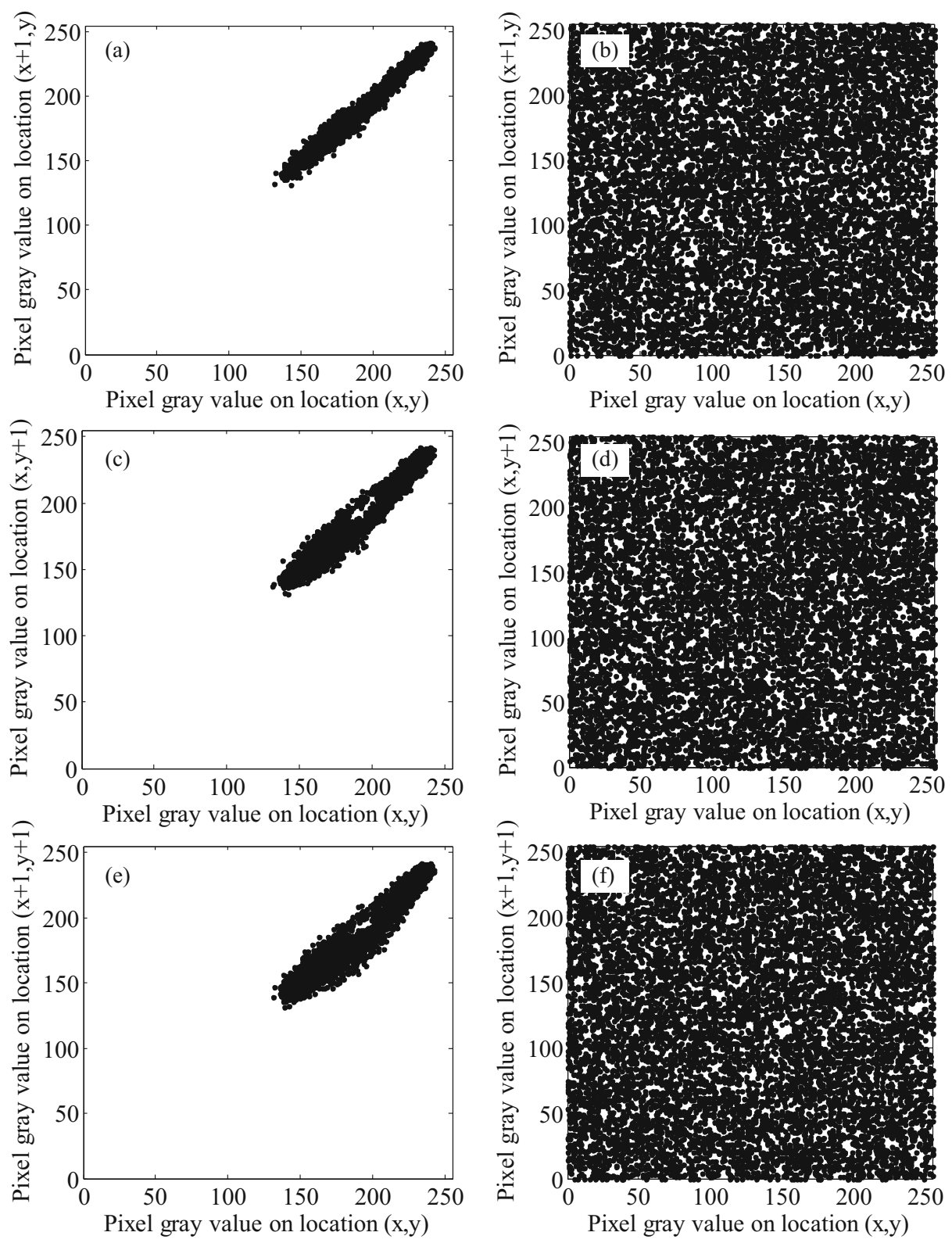

Fig. 11 Distributions of two adjacent pixels in the plain image and in the cipher image 
Table 4 Correlation coefficients of plain and cipher images

\begin{tabular}{|c|c|c|c|c|c|c|}
\hline \multirow[t]{3}{*}{ Image } & \multicolumn{6}{|c|}{ Direction of correlation coefficient } \\
\hline & \multicolumn{2}{|c|}{ Horizontal } & \multicolumn{2}{|l|}{ Vertical } & \multicolumn{2}{|c|}{ Diagonal } \\
\hline & Plain & Cipher & Plain & Cipher & Plain & Cipher \\
\hline Lena & 0.9712 & 0.0046 & 0.9783 & 0.0043 & 0.9547 & -0.0006 \\
\hline Baboon & 0.9437 & 0.0017 & 0.9212 & -0.0036 & 0.9002 & 0.0029 \\
\hline Peppers & 0.9654 & -0.0010 & 0.9552 & 0.0042 & 0.9142 & 0.0025 \\
\hline
\end{tabular}

while those of the cipher image are close to 0 and the values are similar. Therefore, the proposed algorithm can effectively remove the correlations among adjacent pixels in the plain image, and it is strong enough to resist statistical attacks. The results are compared to those of other schemes in Table 5, and the comparison results further prove that the proposed algorithm is better.

\subsection{Occlusion and noise attack analysis}

Occlusion-attack analysis is very important for testing the robustness of the cryptosystem [11]. As for occlusion attack, we are known that the cipher image is partially damaged during the transmission process, and the larger the data loss, the higher the distortion. If the damaged cipher image can be recovered successfully, the encryption scheme would be considered to have the ability to defend against the occlusion attack [23]. Figure 12 (a)-(h) shows different occluded cipher images, in which 100 columns or 100 rows are destroyed in Fig. 12 (a)-(d), $150 \times 150$ pixels from the four corners of the cipher-image and $212 \times 212$ pixels selected from the centre of the cipher-image are destroyed shown in Fig. 12 (e) and Fig. 12 (f), respectively. After decryption, we obtain their corresponding decrypted images shown in Fig. 12 (m)-(n). From the decrypted results, it can be seen that the decrypted images can clearly reflect the information of the original image despite of the existing occlusion attack. To further test the robustness of the algorithm, the cipher-image is further occluded with $75 \%$ in the left and right of the image shown in Fig. $12(\mathrm{~g})$, and the decrypted imaged shown in Fig. 12 (o). It can be seen that the decrypted images can still be clearly recognized

Table 5 Comparative study of the average correlation coefficients of the proposed algorithm $v s$. some existing approaches

\begin{tabular}{lrrr}
\hline Algorithms & \multicolumn{2}{l}{ Direction of correlation coefficient } & Diagonal \\
\cline { 2 - 4 } & Horizontal & Vertical & -0.0018 \\
\hline This work & 0.0021 & 0.0036 & 0.0625 \\
Ref. [15] & 0.0265 & 0.0792 & 0.0029 \\
Ref. [20] & 0.0064 & 0.0078 & 0.0078 \\
Ref. [25] & -0.0046 & 0.0010 & 0.0310 \\
Ref. [48] & 0.0113 & -0.0013 & -0.0012 \\
Ref. [1] & -0.0126 & -0.0050 & \\
\hline
\end{tabular}



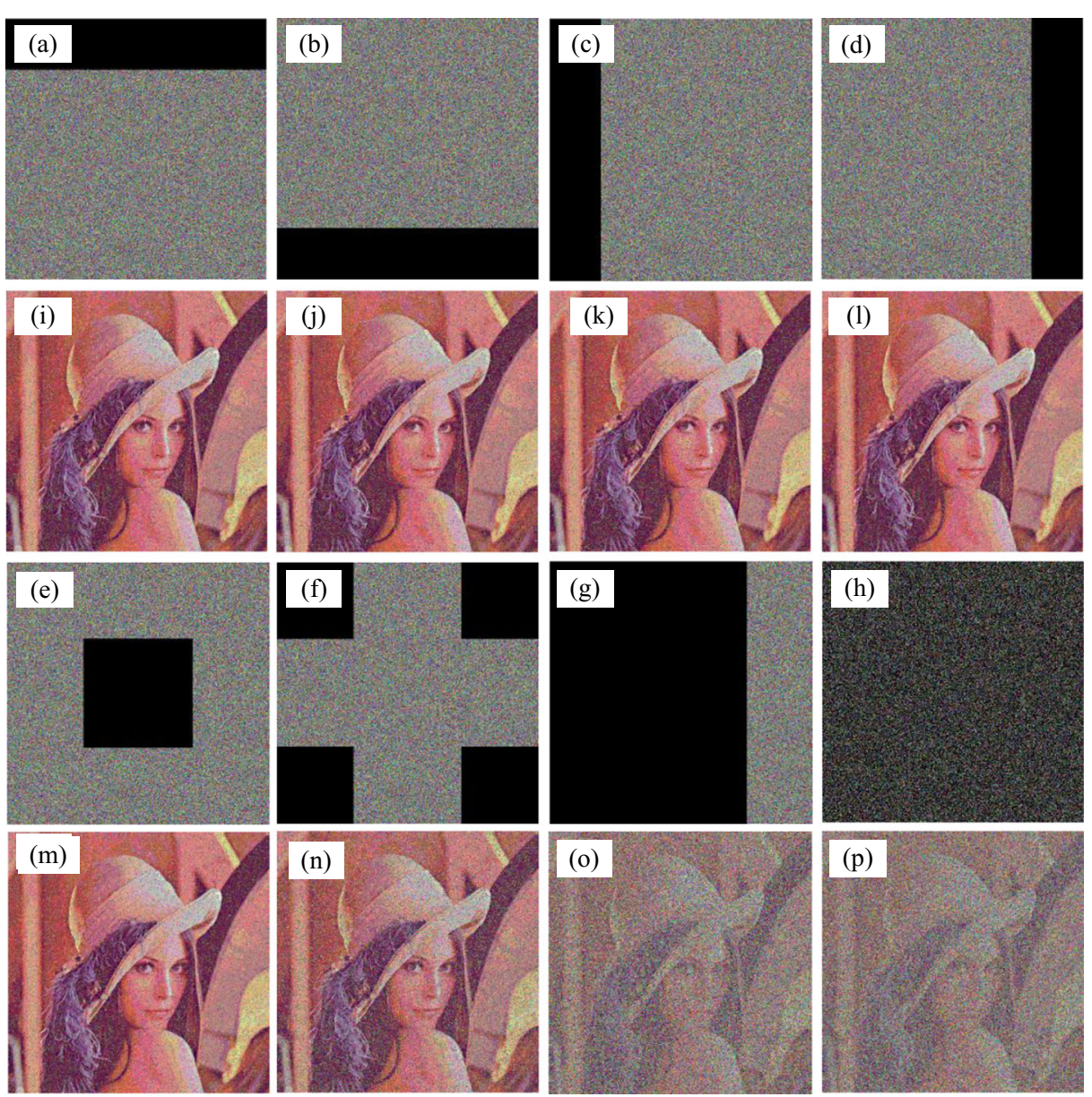

Fig. 12 The decrypted images under different occlusion attacks

even though a strong occlusion attack has occurred. However, occlusion attacks on the image-signal transmission usually happen random. Therefore, the cipher-image is randomly occlude with $80 \%$ shown in Fig. 12 (h), and the decrypted image shown in Fig. 12 (p) can be clearly recognized as well. These results prove that the proposed cryptosystem has sufficient capability to resist occlusion attacks.

On the other hand, the Gaussian noise and Salt and pepper noise are two common noises to be experimented to test the noise attack, and the experimental results are shown in Figs. 13 and 14, respectively. The results demonstrate that the proposed scheme has a strong robustness to resist against the external interference, such as occlusion attack and noise attack.

\subsection{Chosen/known plain-image attacks}

In our algorithm, we use the following strategies to effectively avoid chosen/known plain-image attacks. (a) In the confusion process, we use the novel RCS algorithm to 

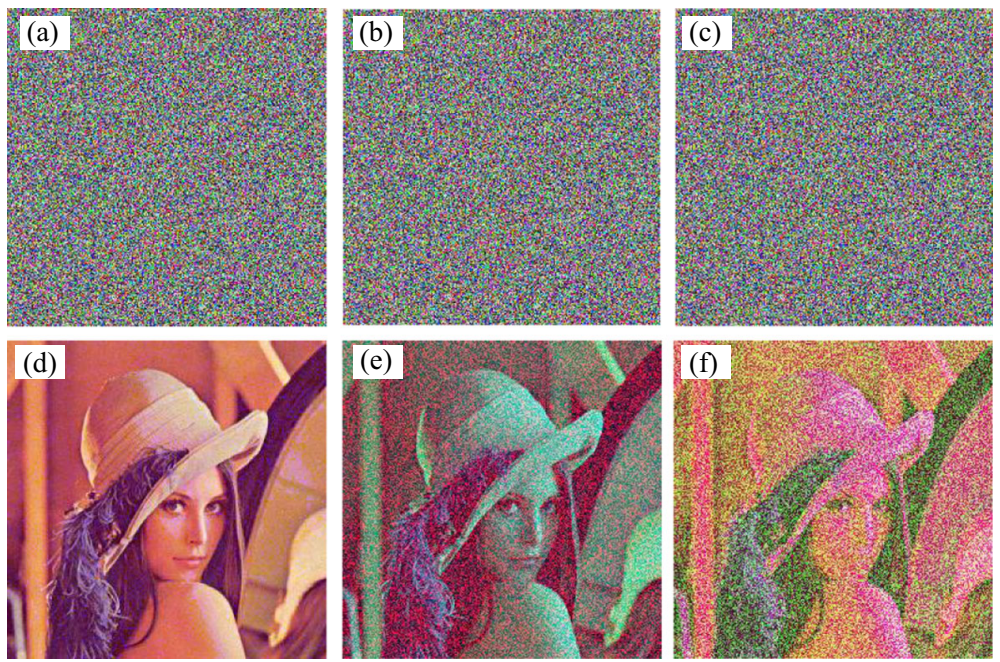

Fig. 13 Gaussian noise attack analysis: (a)-(c) cipher-images with different levels of noise Mean $=0$, Variance = 0.05 ; Mean $=0$, Variance $=0.15$; and Mean $=0$, Variance $=0.25 ;$ (d)-(f) the corresponding decrypted images

permute the image. The image rotation angle and the number of pixel cycle-shifts are stochastically decided by chaotic sequences, so the process exhibits strong randomness and robustness; (b) The confusion and diffusion processes can be realized at the same time. Therefore, the proposed algorithm can resist a divide-and-conquer attack [25]; and (c) The initial keys are closely related to the plain image, as discussed in section 2.1; the secret keys are completely different for different plain images. Hence, our algorithm has enough ability to resist known plain-image attacks.
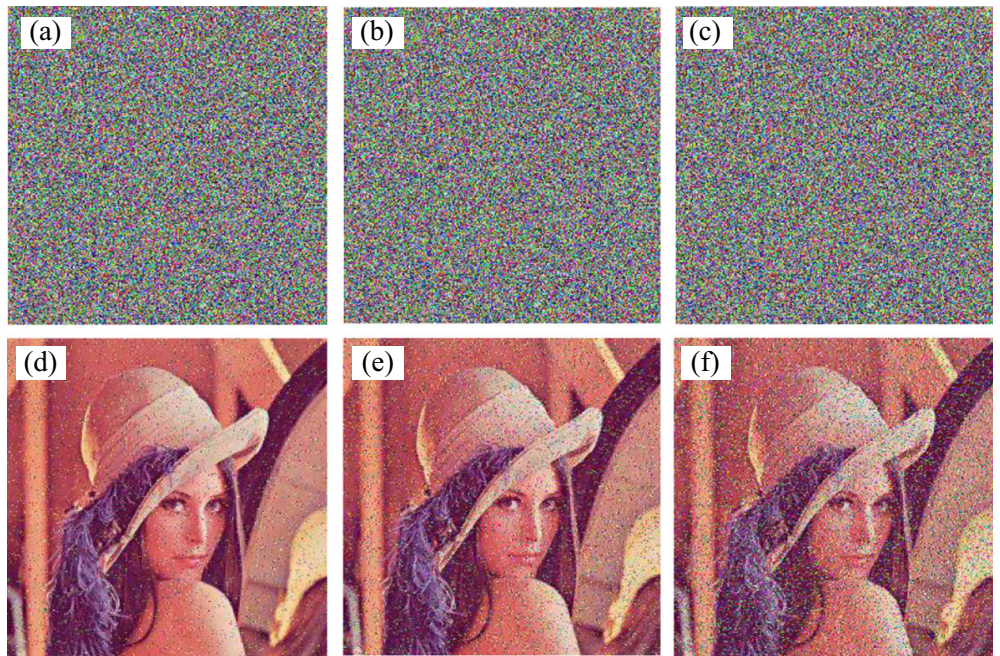

Fig. 14 Salt and pepper noise attack analysis: (a)-(c) cipher-images with Noise density $=5 \%$, Noise density $=$ $10 \%$, and Noise density $=20 \%$, and (d)-(f) the corresponding decrypted images 
Table 6 Execution times of the proposed system (unit: s)

\begin{tabular}{lllllll}
\hline Process & $\begin{array}{l}\text { The quantification } \\
\text { process }\end{array}$ & $\begin{array}{l}\text { The RSC } \\
\text { process }\end{array}$ & $\begin{array}{l}\text { Int2Bin } \\
\text { Converter }\end{array}$ & $\begin{array}{l}\text { Bit-plane XOR } \\
\text { process }\end{array}$ & $\begin{array}{l}\text { Bin2Int } \\
\text { Converter }\end{array}$ & Total \\
\hline Time & 0.143392 & 1.468294 & 0.445377 & 1.687679 & 0.467728 & 4.212 \\
\hline
\end{tabular}

\subsection{Speed analysis and comparisons}

In this section, we discuss the speed performances of the proposed algorithm. To evaluate the proposed scheme and comparable algorithms, different sizes of the standard colour image "Lena", namely, $256 \times 256,512 \times 512$, and $1024 \times 1024$, are selected. The software environment is MATLAB 2014a on the Windows 7 system; the hardware environment includes 2GB RAM, a 2.6 GHz Pentium processor, and 2GB memory. The execution is tested 100 times. The mean test time of every step is listed in Table 6, and the pie chart in Fig. 15 shows that the RSC process and the XOR process occupy $35 \%$ and $40 \%$ of the total time, respectively, and the other processes take up $25 \%$ of the total time.

The average encryption time of different size of the colour image and grey image as the Table 7 shows. In addition, Tables 8 and 9 show the results of the speed performance test, and we clearly find that the encryption speed of our algorithm is faster than other algorithms.

Specifically, the proposed quantization scheme is compared with the other methods in Ref. [23] and Ref. [25]. Experimental results show that their quantification time is $0.165513 \mathrm{~s}$ and $0.085639 \mathrm{~s}$, respectively, which shows that the proposed quantization scheme has a relatively good performance because its execution speed is faster than that in Ref. [23] and the plaintext sensitivity is better than that in Ref. [25] although the quantification time is a little higher.

Besides, the permutation method has been compared with other common methods in the previous work [24], i.e., coupled map lattice (CML) [39], ergodic matrix (EM) [36], constructed two ergodic sequences (CTES) [23], and dynamic random growth technique (DRGT) [40], by using the tests of percentage of adjacent pixels count (PAPC), distance between adjacent pixels (DBAP), the fixed point analysis, correlation analysis, and the permutation time analysis to illustrate Rubik's Cube Scheme has a better performance and is more suitable for image encryption.

\section{Total Encryption Time $=4.212 \mathrm{~s}$}

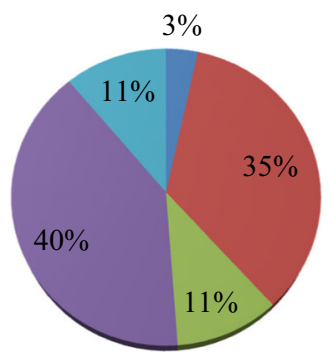

- The quantification process

- The RSC process

- Int2Bin Converter

- Bit-plane XOR process

- Bin2Int Converter

Fig. 15 Average encryption time of each component as a percentage of the total time of the proposed algorithm for "Lena" (size: $256 \times 256$ ) 
Table 7 Average encryption times of the proposed algorithm

\begin{tabular}{cccc}
\hline Image size (pixels) & $256 \times 256$ & $512 \times 512$ & $1024 \times 1024$ \\
\hline Colour image & $4.212(\mathrm{~s})$ & $8.308(\mathrm{~s})$ & $16.254(\mathrm{~s})$ \\
Grey image & $1.318(\mathrm{~s})$ & $2.256(\mathrm{~s})$ & $5.249(\mathrm{~s})$ \\
\hline
\end{tabular}

Table 8 Comparative study of the encryption time of the proposed algorithm $v$. other existing gray image encryption algorithms

\begin{tabular}{clllll}
\hline Image size (pixels) & Our algorithm & Ref. [23] & Ref. [19] & Ref. [13] & Ref. [2] \\
\hline $256 \times 256$ & $1.318(\mathrm{~s})$ & $1.670(\mathrm{~s})$ & $3.704(\mathrm{~s})$ & $3.284(\mathrm{~s})$ & $2.901(\mathrm{~s})$ \\
\hline
\end{tabular}

Table 9 Comparative study of the encryption time of the proposed algorithm vs. other existing color image encryption algorithms

\begin{tabular}{cllll}
\hline Image size (pixels) & Our algorithm & Ref. [38] & Ref. [30] & Ref. [44] \\
\hline $512 \times 512$ & $8.308(\mathrm{~s})$ & $16.170(\mathrm{~s})$ & $8.670(\mathrm{~s})$ & $33.418(\mathrm{~s})$ \\
\hline
\end{tabular}

\section{Conclusion}

In this paper, we proposed an efficient and self-adapting colour-image encryption algorithm based on chaos and interactions among multiple layers. Based on the plain image, a decimal between $(0,1)$ is obtained and quantified to be used as the initial value of the skew tent map and PWLCM map. In the confusion process, a novel RCS algorithm is proposed to achieve inter-scrambling of the three layers. Similarly, the multiple layers are involved in the interencryption of the diffusion process. The experimental results and analyses indicate that the proposed algorithm can not only perform encryption operation but also has high robustness to resist most of known attacks, e.g. statistical analysis, differential attacks and exhaustive attacks, which indicate that the proposed algorithm can be used for digital image encryption.

Acknowledgements This research was supported by the National Natural Science Foundation of China under Grant 61661008, the Guangxi Natural Science Foundation under Grant 2017GXNSFAA198180, 2015GXNSFBA139256 and 2016GXNSFCA380017, the funding of Overseas 100 Talents Program of Guangxi Higher Education, the Research Project of Guangxi University of China under Grant KY2016YB059, Guangxi Key Lab of Multi-source Information Mining \& Security under Grant MIMS15-07, the Doctoral Research Foundation of Guangxi Normal University, the grant from Guangxi Experiment Centre of Information Science, and the Innovation Project of Guangxi Graduate Education under Grant YCSZ2017055.

\section{References}

1. Abbas NA (2015) Image encryption based on Independent Component Analysis and Arnold's Cat Map. Egypt Informatics J 17(1):139-146

2. Abdullah AH, Enayatifar R, Lee M (2012) A hybrid genetic algorithm and chaotic function model for image encryption. AEU - Int J Electron Commun 66(10):806-816

3. Asari VK, Islam MN, Kong D, Shen X (2014) Multiple-image encryption based on optical wavelet transform and multichannel fractional Fourier transform. Opt Laser Technol 57:343-349 
4. Bao L, Zhou Y (2015) Image encryption: Generating visually meaningful encrypted images. Inf. Sci. (Ny). 324:197-207

5. Belazi A, Abd El-Latif AA, Belghith S (2016) A novel image encryption scheme based on substitutionpermutation network and chaos. Signal Process 128:155-170

6. Blakley GR, Borosh I (1979) Rivest-Shamir-Adleman public key cryptosystems do not always conceal messages. Comput Math with Appl 5(3):169-178

7. Chai X, Chen Y, Broyde L (2015) A novel chaotic image encryption scheme using DNA sequence operations. Opt Lasers Eng 73:53-61

8. Chen L, Zhao D (2005) Optical image encryption based on fractional wavelet transform. Opt Commun 254(4-6):361-367

9. Chen G, Mao Y, Chui CK (2004) A symmetric image encryption scheme based on 3D chaotic cat maps. Chaos, Solitons and Fractals 21(3):749-761

10. Chen J, Zhu Z, Fu C, Zhang L, Zhang Y (2015) An efficient image encryption scheme using lookup tablebased confusion and diffusion. Nonlinear Dyn. 81(3):1151-1166

11. Chen J, Zhu Z, Fu C, Zhang L, Yu H (2015) Analysis and improvement of a double-image encryption scheme using pixel scrambling technique in gyrator domains. Opt Lasers Eng 66:1-9

12. El Assad S, Farajallah M (2015) A new chaos-based image encryption system. Signal Process Image Commun 41:1-14

13. Enayatifar R, Abdullah AH, Isnin IF (2014) Chaos-based image encryption using a hybrid genetic algorithm and a DNA sequence. Opt Lasers Eng 56:83-93

14. Gu G, Ling J (2014) Optik A fast image encryption method by using chaotic 3D cat maps. Opt - Int J Light Electron Opt 125(17):4700-4705

15. Guesmi R, Farah MAB, Kachouri A, Samet M (2016) A novel chaos-based image encryption using DNA sequence operation and Secure Hash Algorithm SHA-2. Nonlinear Dyn. 83(3):1123-1136

16. Han F, Zhu C (2011) An Novel Chaotic Image Encryption Algorithm based on Tangent-Delay Ellipse Reflecting Cavity Map System. Procedia Eng 23:186-191

17. Khade P, Narnaware M (2012) 3D Chaotic Functions for Image Encryption. IJCSI Int J Comput Sci Issues 9(3):323-328

18. Li C, Zhang L, Ou R, Wong K, Shu S (2012) Breaking a novel colour image encryption algorithm based on chaos. Nonlinear Dyn. 70(4):2383-2388

19. Lian S, Sun J, Wang Z (2005) A block cipher based on a suitable use of the chaotic standard map. Chaos, Solitons and Fractals 26(1):117-129

20. Liao X, Lai S, Zhou Q (2010) A novel image encryption algorithm based on self-adaptive wave transmission. Signal Process 90(9):2714-2722

21. Liu H, Liu Y (2014) Security assessment on block-Cat-map based permutation applied to image encryption scheme. Opt Laser Technol 56:313-316

22. Luo Y, Du M, Liu J (2014) A symmetrical image encryption scheme in wavelet and time domain. Commun Nonlinear Sci Numer Simul 20(2):447-460

23. Luo Y, Cao L, Qiu S, Lin H, Harkin J, Liu J (2016) A chaotic map-control-based and the plain image-related cryptosystem. Nonlinear Dyn. 83(4):2293-2310

24. Luo Y et al. (2017) A Chaos-based Self-adapting RGB Image Permutation Scheme, in 2017 13th International Conference on Natural Computation, Fuzzy Systems and Knowledge Discovery, 320-325

25. Murillo-Escobar MA, Cruz-Hernández C, Abundiz-Pérez F, López-Gutiérrez RM, Acosta-Del-Campo OR (2015) A RGB image encryption algorithm based on total plain image characteristics and chaos. Signal Process 109:119-131

26. Pareek NK, Patidar V, Sud KK (2011) Colour Image Encryption Scheme Based on Permutation and Substitution Techniques. in 1st International Conference on Computer Science and Information Technology 131:413-427

27. Som S, Dutta S, Singha R, Kotal A, Palit S (2015) Confusion and diffusion of color images with multiple chaotic maps and chaos-based pseudorandom binary number generator. Nonlinear Dyn. 80(1-2):615-627

28. Tong X (2013) Design of an image encryption scheme based on a multiple chaotic map. Commun Nonlinear Sci Numer Simul 18(7):1725-1733

29. Tong X, Liu Y, Zhang M, Xu H, Wang Z (2015) An Image Encryption Scheme Based on Hyperchaotic Rabinovich and Exponential Chaos Maps. Entropy 17(1):181-196

30. Tong X, Zhang M, Wang Z, Ma J (2016) A joint color image encryption and compression scheme based on hyper-chaotic system. Nonlinear Dyn. 84(4):2333-2356

31. Wang X, Jin C (2012) Image encryption using Game of Life permutation and PWLCM chaotic system. Opt Commun 285(4):412-417

32. Wang X, Wang Q (2013) A novel image encryption algorithm based on dynamic S-boxes constructed by chaos. Nonlinear Dyn. 75(3):567-576 
33. Wang X, Zhang H (2015) A color image encryption with heterogeneous bit-permutation and correlated chaos. Opt Commun 342:51-60

34. Wang X, Zhang H (2016) A novel image encryption algorithm based on genetic recombination and hyperchaotic systems. Nonlinear Dyn 83(1-2):333-346

35. Wang K, Pei W, Zou L, Song A, He Z (2005) On the security of 3D Cat map based symmetric image encryption scheme. Phys Lett A 343(6):432-439

36. Wang X, Teng L, Qin X (2012) A novel colour image encryption algorithm based on chaos. Signal Process 92(4):1101-1108

37. Wang $\mathrm{X}$ et al (2014) Analysis and improvement of a chaos-based symmetric image encryption scheme using a bit-level permutation. Commun Nonlinear Sci Numer Simul 77(3):36-50

38. Wang Y, Quan C, Tay CJ (2015) Optical color image encryption without information disclosure using phase-truncated Fresnel transform and a random amplitude mask. Opt Commun 344:147-155

39. Wang X, Zhang Y, Bao X (2015) A novel chaotic image encryption scheme using DNA sequence operations. Opt Lasers Eng 73:53-61

40. Wang X, Liu L, Zhang Y (2015) A novel chaotic block image encryption algorithm based on dynamic random growth technique. Opt Lasers Eng 66:10-18

41. Wang L, Song H, Liu P (2016) A novel hybrid color image encryption algorithm using two complex chaotic systems. Opt Lasers Eng 77:118-125

42. Wang $\mathrm{X}$, Liu $\mathrm{C}, \mathrm{Xu} \mathrm{D}$, Liu $\mathrm{C}$ (2016) Image encryption scheme using chaos and simulated annealing algorithm. Nonlinear Dyn. 84(3):1417-1429

43. Wang X, Liu C, Zhang H (2016) An effective and fast image encryption algorithm based on Chaos and interweaving of ranks. Nonlinear Dyn. 84(3):1595-1607

44. Wu J, Liao X, Yang B (2017) Color image encryption based on chaotic systems and elliptic curve ElGamal scheme. Signal Process 141:109-124

45. Zhang Y, Xiao D (2014) An image encryption scheme based on rotation matrix bit-level permutation and block diffusion. Commun Nonlinear Sci Numer Simul 19(1):74-82

46. Zhang W, Wong K, Yu H, Zhu Z (2012) An image encryption scheme using lightweight bit-level confusion and cascade cross circular diffusion. Opt Commun 285(9):2343-2354

47. Zhang W, Yu H, Zhu Z (2015) Color image encryption based on paired interpermuting planes. Opt Commun 338:199-208

48. Zhang W, Yu H, Zhao Y, Zhu Z (2016) Image encryption based on three-dimensional bit matrix permutation. Signal Process 118:36-50

49. Zhang X, Fan X, Wang J, Zhao Z (2016) A chaos-based image encryption scheme using 2D rectangular transform and dependent substitution. Multimed Tools Appl 75(4):1745-1763

50. Zhu Z, Zhang W, Wong K, Yu H (2011) A chaos-based symmetric image encryption scheme using a bitlevel permutation. Inf Sci (Ny) 181(6):1171-1186

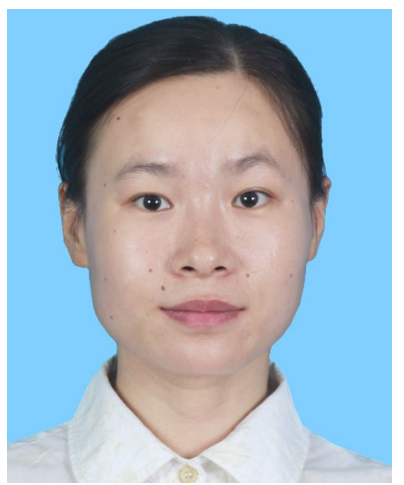

Yuling Luo received her Ph.D. degree in information and communication engineering from South China University of Technology, Guangzhou China, in 2013. She is currently an Associate Professor, Head of School of Electronic Information Engineering, at Guangxi Normal University, Guilin, China. Her research interest includes information security, image processing, chaos theory, artificial intelligence, and embedded system implementation and optimization. 


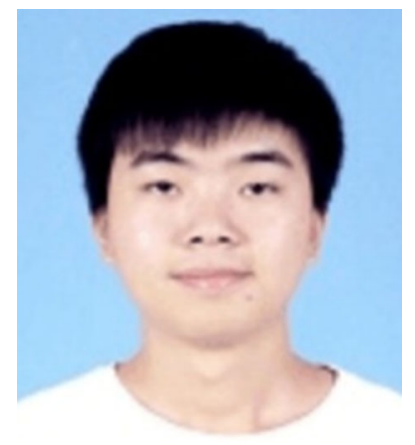

Ronglong Zhou received the B.Eng. degree in Electronics and Information engineering from Heilongjiang University of Finance and Economics, China, in 2015. He is currently a master student in Faculty of Electronic Engineering, Guangxi Normal University. His research interests include multimedia security and nonlinear dynamical system.

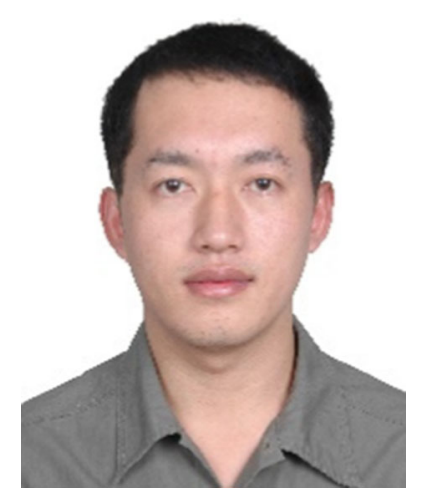

Junxiu Liu received the PhD degree from University of Ulster, UK, with the support of a Vice-Chancellor's Research Scholarship. His research interests relate to: neural glial system, and $\mathrm{SoC} / \mathrm{NoC}$ embedded systems. 


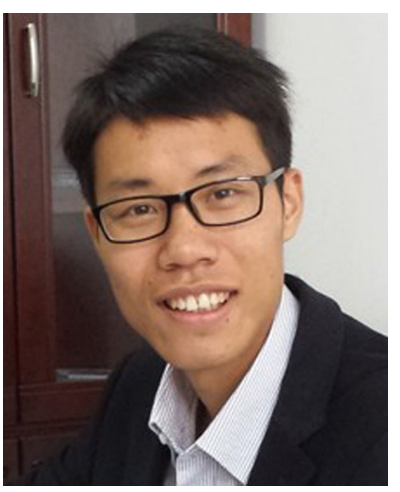

Senhui Qiu is a Lecturer at Guangxi Normal University. His research includes applied science, and embedded system design.

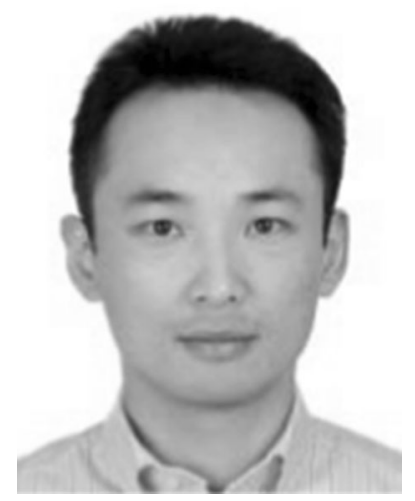

Yi Cao received the B.Eng degree in navigation and control in aeronautics from Beihang University, Beijing, China in 2002, and M.S. degree in computer science from Florida International University, Miami, FL, USA, in 2005, and the Ph.D. degree in financial machine learning from the Ulster University, Londonderry, UK, in 2015. He was an Integrated Circuit and Hardware Engineer with Vimicro, Beijing, and Conexant Beijing Design Centre, Beijing, from 2005 to 2008. From 2008 to 2011, he was a Senior System Engineer with Ericsson, Beijing. He is currently a Lecturer at University of Surrey, Surrey, UK. 